\title{
CHARACTERISTIC RESONANCE FEATURES OF SOI-CMOS-COMPATIBLE SILICON NANOELECTROMECHANICAL DOUBLY-CLAMPED BEAMS UP TO $330 \mathrm{MHZ}$
}

Yoshishige Tsuchiya ${ }^{1}$, Yilin Feng ${ }^{1}$, Christos Giotis ${ }^{1}$, Naoaki Harada ${ }^{1,2}$, Mitsuhiro Shikida ${ }^{2}$, Cécilia Dupré $^{3}$, Eric Ollier ${ }^{3}$, Faezeh Arab Hassani ${ }^{1,4}$, and Hiroshi Mizuta ${ }^{1,5}$

${ }^{1}$ Department of Electronics and Computer Science, University of Southampton, UK ${ }^{2}$ Department of Biomedical Information Sciences, Hiroshima City University, JAPAN

${ }^{3}$ CEA-Leti-MINATEC, FRANCE

${ }^{4}$ Department of Electrical and Computer Engineering \& Singapore Institute for Neurotechnology,

National University of Singapore, SINGAPORE

${ }^{5}$ School of Material Sciences, Japan Advanced Institute of Science and Technology, JAPAN

\begin{abstract}
This paper reports novel characteristic features of thermally-passivated $\mathrm{Si}$ nanoelectromechanical (NEM) beams fabricated via SOI-CMOS compatible processes with top-down hybrid EB/DUV lithography. Considerable difference of the resonance frequencies between the measurement results of the NEM beams with various lengths and the finite element simulation results suggests that effects of the undercut of the beam supports are serious for sub-micron beams. The resonance frequency of 332.57 $\mathrm{MHz}$ observed for an 800-nm-long beam is, to our knowledge, the highest ever as the fundamental resonance mode of lithographically-defined Si NEM beams. Clear change of the temperature dependence of the resonance frequencies with the varied beam lengths, observed for the first time, can be explained by considering effects of thermally-induced strain on the longer beams at higher temperatures.
\end{abstract}

\section{INTRODUCTION}

Nanoelectromechanical (NEM) resonators have been attracting much attention not only for their potential applications for ultrasmall mass sensors [1] and nano radio [2], but also for investigating fundamental physics [3]. While using bottom-up-grown carbon nanotubes $[4,5]$ or $\mathrm{Si}$ nanowires $[6,7]$ is beneficial for reducing the diameter of the beam, top-down-fabricated silicon beams are advantageous for integration with $\mathrm{Si}$ CMOS-based technology [8-11]. We have designed and fabricated doubly-clamped Si NEM resonators with various beam dimensions via SOI-CMOS compatible technology, and fundamental resonance characteristics of the devices have been reported $[12,13]$. This paper will show the results of systematic measurements of the beams under varied bias conditions and will compare with the simulation results of the frequency-dependent behavior of the beam displacement. Temperature dependence of the resonance will be also studied for in-depth understanding of the characteristic resonance features of the NEM beams whose length is scaled down to $1 \mu \mathrm{m}$.

\section{EXPERIMENTAL}

Figure 1 schematically shows the device fabrication process of NEM resonators used in this study. An n-type heavily-doped SOI layer with the doping concentration of $10^{19} \mathrm{~cm}^{3}$ (Fig. 1(a)) was patterned (Fig. 1(b)) and then the
BOX layer was etched to make the SOI beams suspended (Fig. 1(c)). The surface of the beams was thermally oxidized to passivate with high-quality oxide (Fig. 1(d)). Subsequent Poly-Si deposition (Fig. 1(e)) was followed by interlayer $\mathrm{SiO}_{2}$ deposition, contact hole etching (Fig. 1(f)). After metallization, an $\mathrm{H}$-shape pattern was transferred to etch the interlayer oxide (Fig. 1(g)) and then the sacrificial Poly-Si layer was etched to form thermally-passivated suspended silicon beams (Fig. 1(h)).
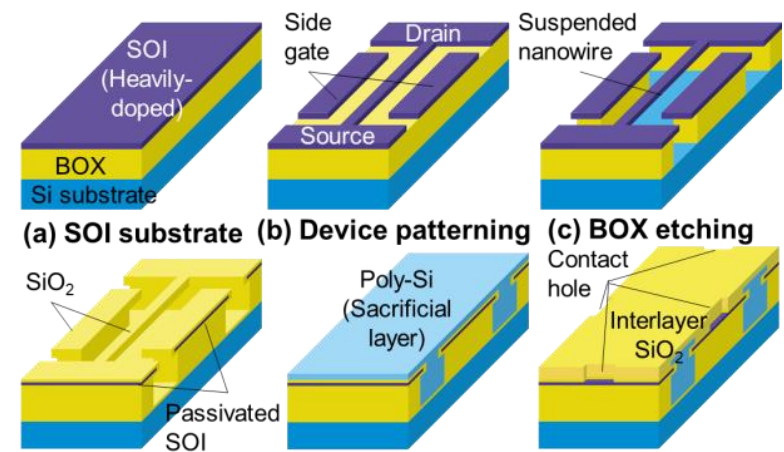

(d) Therma
oxidation
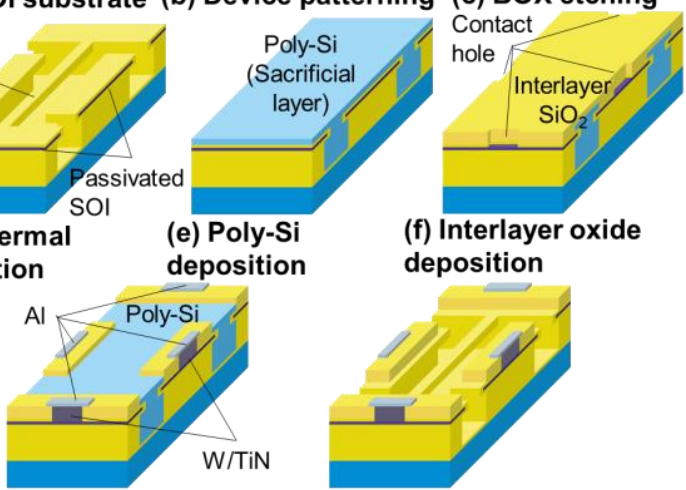

(f) Interlayer oxide

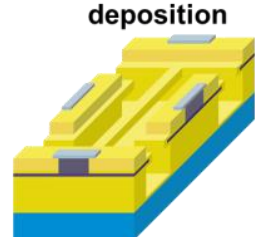

(g) Metallisation \& $\mathrm{SiO}_{2}$ etching (h) Poly-Si etching

Figure 1: A schematic diagram of the fabrication process flow $((a)-(h))$ of surface-passivated Si NEM resonators via SOI-CMOS compatible processes.

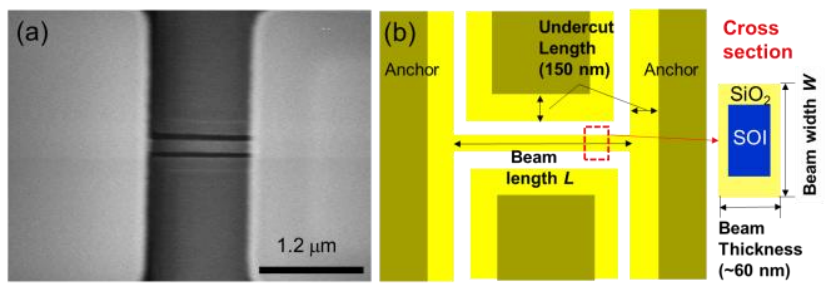

Figure 2:(a) An SEM image and (b) schematic description of the suspended beam.

An SEM image in Fig. 2 (a) confirms successful beam formation. As a result of using the isotropic etching to make the beams suspended, the edges of the anchors are 
also undercut as schematically shown in Fig. 2 (b). The undercut length of $\sim 150 \mathrm{~nm}$ can be identified from the SEM image in Fig. 2 (a). About 14-nm-thick $\mathrm{SiO}_{2}$ is grown around the beams under the thermal oxidation condition used in this process, resulting in a core-shell cross section depicted in Fig. 2 (b). Therefore, the designed beam width $W_{\mathrm{d}}$ and the actual beam width $W$ are different. The dimensions of the beams are summarized in Table 1.

Table 1: Summary of the beam length and width of the samples used in this study.

\begin{tabular}{|r|r|r|r|}
\hline Sample & $L(\mu \mathrm{m})$ & \multicolumn{1}{l|}{$W_{\mathrm{d}}(\mathrm{nm})$} & \multicolumn{1}{l|}{$W(\mathrm{~nm})$} \\
\hline A & 2 & 105 & 121 \\
\hline B & 2 & 75 & 91 \\
\hline C & 1.5 & 105 & 121 \\
\hline D & 1.5 & 75 & 91 \\
\hline E & 1 & 105 & 121 \\
\hline F & 1 & 75 & 91 \\
\hline G & 0.8 & 105 & 121 \\
\hline
\end{tabular}

A frequency modulation (FM) detection method [5,7] was used to measure the resonance of the NEM resonators. The RF signal generated by the Rohde \& Schwartz SMJ100A generator was modulated by the reference signal of $2 \mathrm{kHz}$ from the SRS SR830 lock-in amplifier. The mechanical oscillation was activated under the DC bias $V_{\mathrm{g}}$ applied to the back gate by using Agilent B1500 and the enhanced current at the resonance frequency was detected by the lock-in amplifier. A schematic of the measurement system is shown in Fig. 3. The samples were placed on the temperature-controllable stage in a vacuum chamber with the electrical probes equipped (LakeShore EMTTP4).

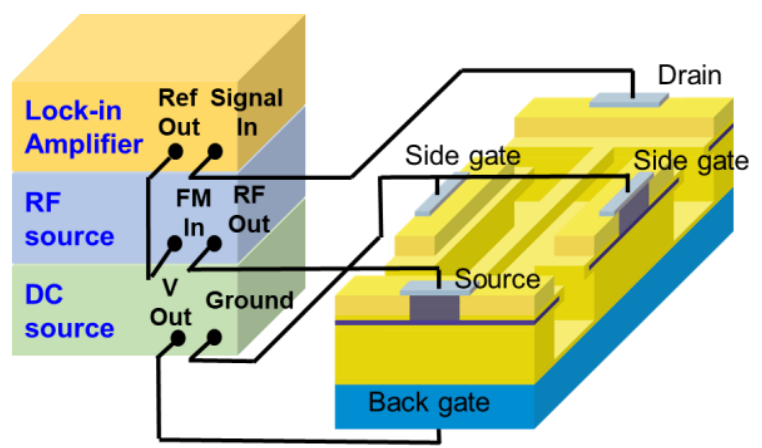

Figure 3: A schematic diagram of the FM detection set up for resonance measurements. The side gates were not used.

\section{RESULTS AND DISCUSSION}

Figure 4 (a) shows an in-phase current, $X$, measured in the lock-in amplifier as a function of the applied RF frequency, $f$, for Sample A of $L=2 \mu \mathrm{m}$ and $W_{\mathrm{d}}=105 \mathrm{~nm}$. The peak has emerged around $96 \mathrm{MHz}$ with increasing the back gate voltage $V_{\mathrm{g}}$. The observed peaks correspond to mechanical resonance as $X$ is known to be proportional to the frequency derivative of the displacement of the beam [5]. A clear nonlinear behavior at the higher gate voltages is a characteristic feature of Duffing oscillators which is observed typically in electromechanical resonance. Figure 4 (b) shows a resonance peak fitted by the equation [5],
$X(f)=A \frac{2 f\left(f^{2}-f_{0}^{2}-f_{0}^{2} / Q\right)\left(f^{2}-f_{0}^{2}+f_{0}^{2} / Q\right)}{\left[\left(f_{0}^{2}-f^{2}\right)^{2}+\left(f_{0} f / Q\right)^{2}\right]^{2}}+B$,

where $f_{0}$ is the resonance frequency, $Q$ is the quality factor of the resonance, and $A$ and $B$ are a scaling factor and an offset for the in-phase current $X$, respectively. The $f_{0}$ and $Q$ extracted by fitting the curves in Fig. 4 (a) are plotted in Fig. 4 (c) as a function of $V_{\mathrm{g}}$. The decrease of the resonance frequency $f_{0}$ is due to softening of the beam under the applied voltage. The $Q$ values are $\sim 600$ and the large increase of $Q$ at the higher gate voltages is due to the asymmetric line shape in the nonlinear regime.
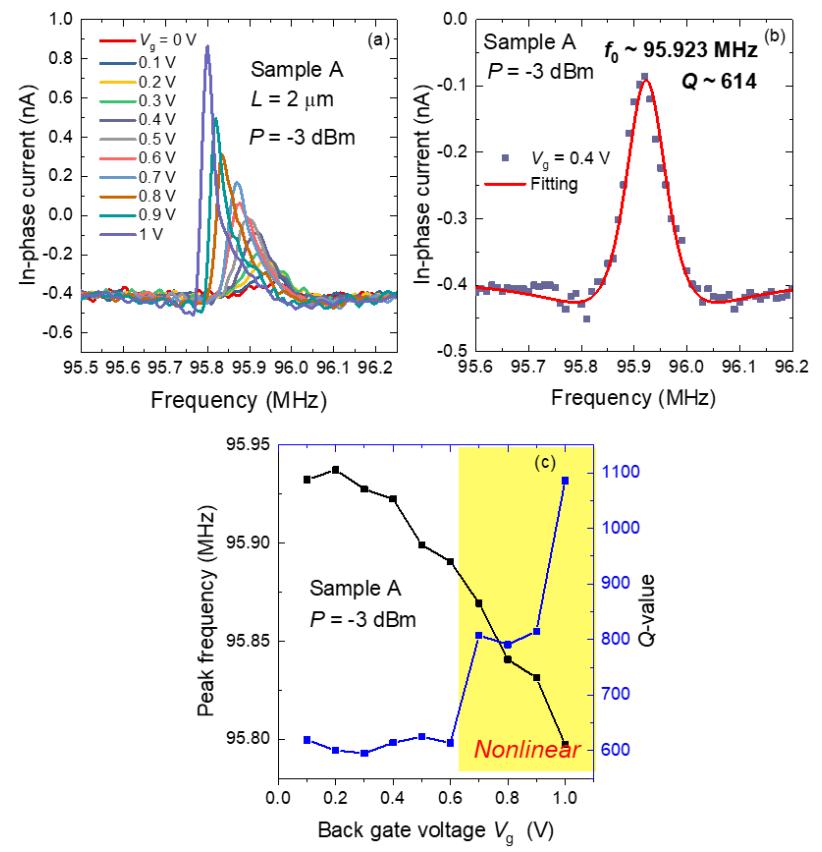

Figure 4: (a) Resonance peaks for Sample A with respect to the back-gate voltage $V_{g}$. (b) The curve fitting results for the peak taken with $V_{g}=0.4 \mathrm{~V}$. (c) Peak frequency and $Q$-value with respect to $V_{g}$. Nonlinear effects are significant in the hatched region.

Figure 5 summarizes how the resonance is changed depending on the beam length $L$, designed beam width $W_{\mathrm{d}}$, and applied voltage $V_{\mathrm{g}}$. While the resonance frequency $f_{0}$ becomes higher with shorter $L$ (Fig. 5 (a), (c) and (e)), how $f_{0}$ shifts with respect to $W_{\mathrm{d}}$ depends on $L$. The resonance frequency $f_{0}$ for the beams with $L=2$ and $1.5 \mu \mathrm{m}$ shifts higher with the narrower $W_{\mathrm{d}}$ (Fig. 5(a) and (b), and Fig. 5 (c) and (d)), which is consistent with the idea that $f_{0}$ is determined by the mass of the beam. On the other hand, for the beam with $L=1.0 \mu \mathrm{m}, f_{0}$ shifts slightly lower with decreasing $W_{\mathrm{d}}$ (Fig. 5(e) and (f)), suggesting the dominant factor to determine $f_{0}$ is not the mass of the beam but the mechanical stiffness.

Finite element analysis (FEA) of the models of the NEM resonators have been also conducted to compare with the experimental observation. A commercial FEA software Comsol Multiphysics [14] is used for simulations. For the frequency domain analysis, the RF drive signal is added as a harmonic perturbation in addition to the DC offset voltage. Figure 6 (a) - (d) show the calculated frequency dependence of the displacement field $D_{\mathrm{z}}$ for the models with $L=2,1.5,1.0$ and $0.8 \mu \mathrm{m}$, respectively. The undercut 
length of $150 \mathrm{~nm}$ was considered in the simulations and clear anomalies in $D_{z}$ corresponds to the resonance. The frequency where the anomaly is observed, $f_{\mathrm{s}}$, is generally higher than $f_{0}$ extracted from the experiments.
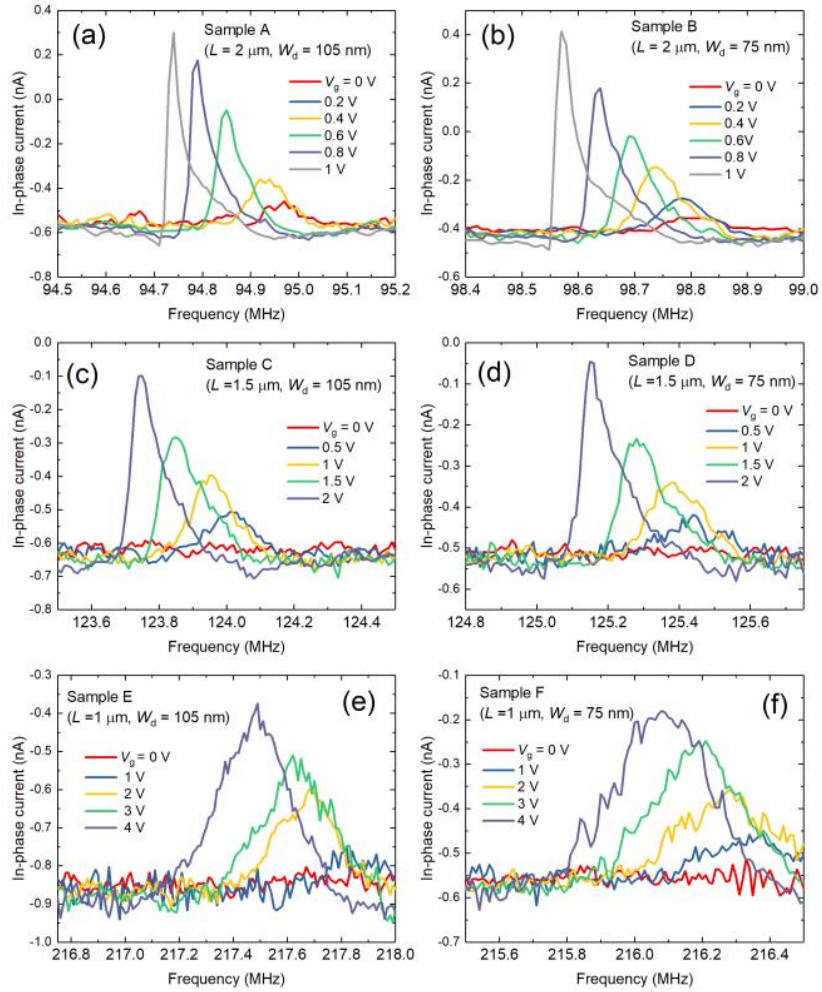

Figure 5: Observed resonance peaks with respect to the appropriate gate voltages for (a) Sample $A,(b) B,(c) C,(d)$ $D$, (e) $E$, and $(f) F$, respectively.
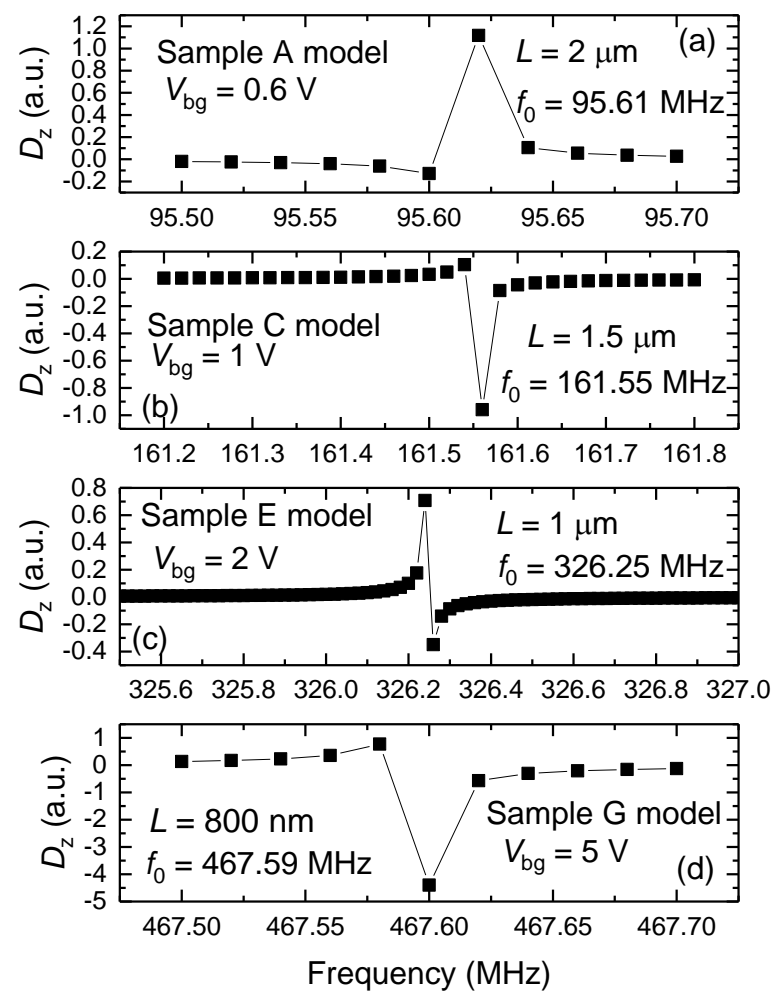

Figure 6: (a) Calculated displacement $D_{z}$ as a function of the frequency for the models of (a) Sample A, (b) $C,(c) E$, and $(d) G$, respectively.
Regarding Sample G with $L=800 \mathrm{~nm}$, we have measured the $X(f)$ under the condition with $V_{\mathrm{g}}$ floated and with a higher RF power, and observed a resonance peak at $332.57 \mathrm{MHz}$ with the $Q$-value of $\sim 731$ as shown in Fig. 7 .

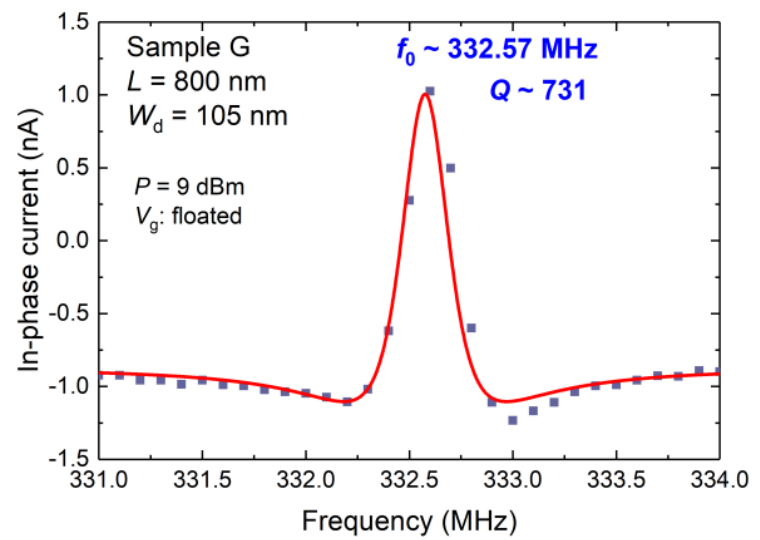

Figure 7: A resonance peak observed for Sample $G$ with $L$ $=800 \mathrm{~nm}$.

Table 2 summarizes the $f_{\mathrm{s}}$ and $f_{0}$ for the samples with the different beam lengths, together with the modal analysis results, $f_{\mathrm{m}}$ for the beams without considering the suspended anchors. The discrepancy between $f_{\mathrm{m}}$ and $f_{\mathrm{s}}$ is reasonably explained due to lack of considering the undercut of the anchors. On the other hand, the discrepancy between $f_{\mathrm{s}}$ and $f_{0}$ indicates that consideration of the undercut effects is not enough to explain the experimental observation. Further accurate consideration of the geometry of the suspended object and boundary conditions would be essential in modelling the resonators scaled down to sub-microns.

Table 2: Summary of the resonance frequencies: $f_{m}$ by modal analysis of beams, $f_{s}$ by FEM calculation considering undercut, and fo extracted from measurement results.

\begin{tabular}{|r|r|r|r|}
\hline \multicolumn{1}{l|}{$L(\mu \mathrm{m})$} & $f_{\mathrm{m}}(\mathrm{MHz})$ & \multicolumn{1}{|l|}{$f_{\mathrm{s}}(\mathrm{MHz})$} & $f_{0}(\mathrm{MHz})$ \\
\hline 2.0 & 98.9 & 95.61 & 94.85 \\
\hline 1.5 & 176 & 161.6 & 124.0 \\
\hline 1.0 & 393 & 326.3 & 217.7 \\
\hline 0.8 & 611 & 467.6 & $(322)$ \\
\hline
\end{tabular}

The temperature dependence of the resonance frequency has been also measured for in-depth understanding of dominant factors that determine the resonance characteristics. Figure 8 (a), (b), and (c) show how the resonance peak position and the line shape are changed with respect to the sample temperatures and the beam lengths. For Sample A with $L=2 \mu \mathrm{m}, f_{0}$ shifts higher with increasing temperature while the trend is opposite for Sample E with $L=1 \mu \mathrm{m}$. Interestingly the $f_{0}$ of Sample C with $L=1.5 \mu \mathrm{m}$ shows an intermediate behavior where $f_{0}$ shifts higher up to $75^{\circ} \mathrm{C}$ and then shifts lower at higher temperature up to $125^{\circ} \mathrm{C}$. How the resonance frequencies are changed with respect to the temperature is summarized in Fig. 8 (d) - (f). The results strongly suggest that there are 
competing factors to determine the resonance frequency of the scaled NEM beams. A possible explanation is that thermally-induced strain could make the longer beam of Sample A stiffer, while the shorter beam of Sample E are softened with increasing temperature. The data of Sample $\mathrm{C}$ indicates the characteristic length scale where a crossover of these two mechanisms takes place is around $L$ $=1.5 \mu \mathrm{m}$.
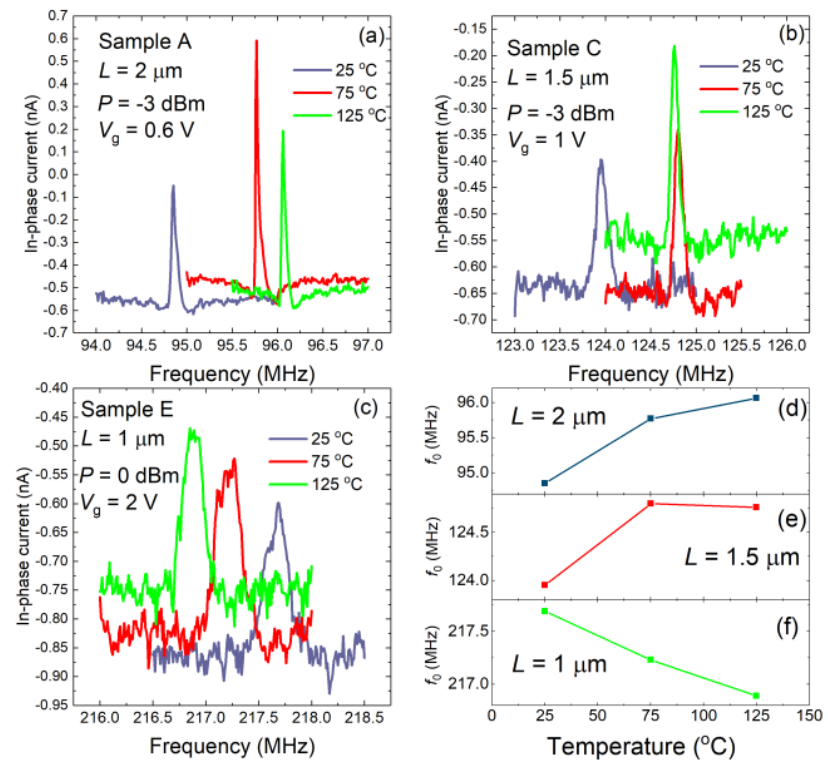

Figure 8: Peak shifts with respect to the temperature for (a) Sample A, (b) $C$, and (c) E and the plots $((d)-(f))$.

\section{CONCLUSIONS}

Resonance characteristics of thermally-passivated $\mathrm{Si}$ nanoelectromechanical (NEM) beams fabricated via top-down SOI-CMOS compatible processes have been systematically studied. Comparison between the measured and simulated resonance frequencies suggests that contribution of the undercut anchors to the resonance characteristics must be seriously taken into account in designing NEM resonators with the beam length of less than $1 \mu \mathrm{m}$. The resonance frequency of $332.57 \mathrm{MHz}$ for the 800-nm-long beam has been successfully observed as the highest as the fundamental resonance mode of the lithographically-defined $\mathrm{Si}$ NEM beams. Effects of thermally-induced strain might be a key to understand the measurement results of the temperature dependence of the resonance frequencies.

\section{ACKNOWLEDGEMENTS}

This work has been partly supported by EU FP7 project NEMSIC (224525), TOBITATE! Young Ambassador Program, MEXT, Japan, and University of Southampton Summer Internship Program.

\section{REFERENCES}

[1] J. Chaste, A. Eichler, J. Moser, G. Ceballos, R. Rurali and A. Bachtold, "A nanomechanical mass sensor with yoctogram resolution", Nature Nanotechnology, vol. 7, pp. 301-304, 2012.

[2] K. Jensen, J. Weldon, H. Garcia, and A. Zettl, "Nanotube Radio", Nano Lett., vol. 7, pp. 3508-3511,
2007.

[3] J. D. Teufel, T. Donner, Dale Li, J. W. Harlow, M. S. Allman, K. Cicak, A. J. Sirois, J. D. Whittaker, K. W. Lehnert and R. W. Simmonds "Sideband cooling of micromechanical motion to the quantum ground state", Nature, vol. 475, pp. 359-363, 2011.

[4] V. Sazonova, Y. Yaish, H. Üstünel, D. Roundy, T. A. Arias and P. L. McEuen "A tunable carbon nanotube electromechanical oscillator", Nature, vol. 431, pp. 284-287, 2004.

[5] V. Gouttenoire, T. Barois, S. Perisanu, J. L. Leclercq, S. T. Purcell, P. Vincent, and A. Ayari, "Digital and FM demodulation of a doubly clamped single-walled carbon-nanotube oscillator: towards a nanotube cell phone", Small, vol. 6, pp. 1060 - 1065, 2010.

[6] R. He, X. L. Feng, M. L. Roukes, and P. Yang, "Self-Transducing Silicon Nanowire Electromechanical Systems at Room Temperature", Nano Lett., vol. 8, pp. 1756-1761, 2008.

[7] M. Sansa, M. Fernández-Regúlez, Á. San Paulo, and F. Pérez-Murano, "Electrical transduction in nanomechanical resonators based on doubly clamped bottom-up silicon nanowires", Appl. Phys. Lett., vol. 101, p. 243115, 2012.

[8] S. T. Bartsch, C. Dupré, E. Ollier and A. M. Ionescu, "Resonant-Body Silicon Nanowire Field Effect Transistor without Junctions", in Proc. IEDM 2012, San Francisco, USA, 10-13 Dec. 2012, pp. 351-354.

[9] E. Ollier, C. Dupré, G. Arndt, J. Arcamone, C. Vizioz, L. Duraffourg, E. Sage, A. Koumela, S. Hentz, G. Cibrario, P. Meininger, K. Benotmane, C. Marcoux, O. Rozeau, G. Billiot, E. Colinet, F. Andrieu, J. Philippe, F. Aussenac, D. Mercier, H. Blanc, T. Ernst and P. Robert, "Ultra-scaled high-frequency single-crystal Si NEMS resonators and their front-end co-integration with CMOS for high sensitivity applications", in Proc. 2012 IEEE MEMS, Paris, France, 29 Jan. - 2 Feb., 2012, pp. 1368-1371.

[10] A. Koumela, S. Hentz, D. Mercier, C. Dupré, E. Ollier, P. X.-L. Feng, S. T. Purcell, and L. Duraffourg, "High frequency top-down junction-less silicon nanowire resonators", Nanotechnology, vol. 24, p. 435203, 2013.

[11]S. T. Bartsch, M. Arp and A. M. Ionescu, "Junctionless Silicon Nanowire Resonator", IEEE J. Electron Dev. Soc., vol. 2, pp. 8-15, 2014.

[12]F. Arab Hassani, Y. Tsuchiya and H. Mizuta, "In-Plane Resonant Nanoelectromechanical Sensors: A Comprehensive Study on Design, Fabrication and Characterization Challenges", Sensors, vol. 13, pp. 9364-9387, 2013.

[13] Y. Tsuchiya, N. Harada, C. Giotis, J. Lamb, F. Arab Hassani, M. Shikida, C. Dupré, E. Ollier, S. T. Bartsch, A. M. Ionescu, and H. Mizuta, "RF characterisation of CMOS-compatible Silicon-onInsulator Nanoelectromechanical Resonators", in Proc. MNE2017, Braga, Portugal, 18-22 Sep. 2017.

[14] Comsol Multiphysics, https://www.comsol.com/

\section{CONTACT}

*Y. Tsuchiya, tel: +44-23-8059-9312;

yt2@ecs.soton.ac.uk 